\title{
Polyamide 6/Layered Silicate Nanocomposites
}

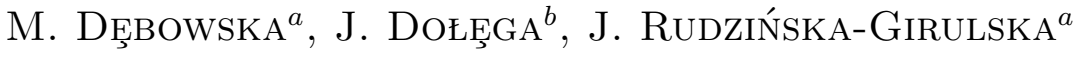 \\ AND J. PigŁOWski ${ }^{b}$ \\ ${ }^{a}$ Institute of Experimental Physics, University of Wrocław \\ pl. Maksa Borna 9, 50-204 Wrocław, Poland \\ ${ }^{b}$ Polymer Engineering and Technology Division, Faculty of Chemistry \\ Wrocław University of Technology \\ Wybrzeże Wyspiańskiego 27, 50-370 Wrocław, Poland \\ Polyamide 6 (PA6) and its two exfoliated nanocomposites (PA6/Nf919 \\ and PA6/BZ-COCO), with bentonite (2.5 wt.\%) organophilically treated \\ with different cations, were studied. Improved mechanical properties, \\ changes in crystallinity and morphology as well as higher glass transition \\ temperature values were observed for the nanocomposites in comparison to \\ the neat PA6. For the nanocomposite PA6/BZ-COCO, of better surface \\ modification of platelets and better interaction between the polymeric ma- \\ trix and the organobentonite, higher values of Young's modulus and yielding \\ point together with higher contribution of larger free volume holes to free \\ volume distributions occurred.
}

PACS numbers: 78.70.Bj, 36.10.Dr, 82.35.Lr, 81.05.Qk

\section{Introduction}

Polymer/layered silicate nanocomposites have attracted much attention over a last decade as novel materials of characteristics that cannot be achieved with the use of inorganic fillers at macro- and microscopic dispersion. After incorporation of small amounts $(\leq 5 \mathrm{wt} . \%)$ of organically modified layered silicate into polymer matrix, significant improvement of chemical, mechanical, and physical properties of the polymer is observed [1]. The reinforcing effect of clay nanoparticles is related to their high aspect ratio and to filler/matrix interactions. Due to the compatibilization, followed by strong interfacial interaction between the two materials, the dispersion of the silicate at nanometer scale is gained. The uppermost enhancement of material properties takes place when exfoliation (delamination) of the silicate happens.

Many properties of polymers and polymer/clay composites as e.g. flow behavior, physical aging, elastic moduli, transport properties are considered to be strongly related to free volume. In Ref. [2] pressure-volume-temperature (PVT) 
dependence was investigated for PA6 and based on it a nanocomposite containing 1.6 wt.\% of exfoliated montmorillonite. Excellent agreement between experiment and the results from the Simha-Somcynsky lattice-hole theory [3] was found. It was concluded that addition of exfoliated clay resulted in a large free volume loss (12-16\% depending on pressure and temperature). The suggestion was given that PA6 chains are adsorbed on solid surface of platelets of montmorillonite. Immobilization of the first few adsorbed layers took probably place. The "hairy clay platelet" (HCP) model was used [2], consistent with the observed reduction of the free volume. According to the model exfoliated clay platelets are considered as solid particles "uniformly covered on all sides by a $6 \mathrm{~nm}$ thick layer of solid- like PA6". Two types of macromolecules were supposed to exist in the matrix; those which form a ca. $100 \mathrm{~nm}$ thick envelope around HCP and others, free of HCP influence. In opinion of authors [2] the free PA6 molecules (of properties of bulk PA6) may occur only in systems with the organoclay content smaller than 1.2 wt.\%.

Positron annihilation lifetime spectroscopy (PALS) is a method that gives an opportunity to estimate sizes of free volume holes from the lifetimes of orthopositronium (o-Ps) [4]. The process of quenching of $o$-Ps by pick-off in the holes results in the lifetime increasing when the dimensions of the holes increase. PALS is a recognized method by people who determine the unoccupied volume in polymers from molecular dynamic simulations. They choose for the simulations such materials which are well characterized, especially those for which there already exist free volume data determined by positron lifetime experiments (as e.g. in [57]). Good qualitative agreement was demonstrated between size distributions of free volume elements in some polymers obtained by molecular computer modeling and experiments using positron annihilation technique [6]. Good correlation was found between PALS average hole size and transport coefficients [8].

\section{Samples}

To our studies polyamide 6, type Tarnamid T-27, was supplied by Zakłady Azotowe Tarnów-Mościce S.A. Polyamide 6, also known as nylon 6, is semicrystalline polymer with two major crystalline forms $(\alpha$ and $\gamma)$ [9]. The $\alpha$-form is thermodynamically stable. It has a monoclinic structure, where neighboring antiparallel chains form hydrogen bonds which in turn generate planar hydrogen bonded sheets (ac-planes). These sheets stack together via van der Waals interaction. The $\gamma$-form also has a monoclinic structure, but because the amide units tilt and rotate out of the $a c$-plane, hydrogen bonds occur between parallel chains. The hydrogen bonding is poorer in the $\gamma$-form compared to the $\alpha$-form.

\subsection{Fillers}

Two fillers were used: bentonite Nf919 and bentonite BZ-COCO. In case of bentonite Nf919 the manufacturer (Süd Chemie A.G.) superseded sodium cations by cationic surfactant: stearylbenzyldimethylammonium chloride. In BZ-COCO 
modification of the bentonite (Bentonit Specjal supplied by Zakłady GórniczoMetalowe Zȩbiec S.A.) was performed [10] with a mixture of 3-(alkylamino)propyltrimethylammonium methylsulphates with different alkyl chain length (COCO) produced by PPC ROKITA. Bentonite is a clay deposit [11], primarily composed of montmorillonite, formed by weathering of volcanic ash. Montmorillonite (layered silicate) is a 2:1 clay mineral which belongs to a group of smectite [12].

\subsection{Preparation of the nanocomposites}

Prior to each processing step PA6 was dried in a vacuum oven at $80^{\circ} \mathrm{C}$ for $24 \mathrm{~h}$, to avoid moisture induced degradation reactions. Nanocomposites of PA6 and two types of fillers were prepared by melt compounding using a twin screw extruder (Maris, $L / D=40$ ). For comparison neat PA6 was also extruded in the extruder at the same processing conditions. The dried pellets were injected to standard specimens for mechanical testing and other studies. Further details of the modification of the bentonite BZ-COCO like the preparation of the nanocomposites can be found in [10].

\section{Properties of the samples}

All the samples have been characterized by many experimental techniques [10]. In the paper (because of its limited volume) only some final results from transmission electron microscopy (TEM), X-ray diffractometry (XRD), differential scanning calorimetry (DSC) and positron annihilation lifetime spectroscopy (PALS) are given. Conditions of TEM, XRD and DSC measurements can be found in [10]. As transmission electron microscope studies showed [10] the exfoliated nanocomposites were obtained, in which particles of the organo-bentonites dispersed in the PA6 matrix into individual layers, having a thickness of 1-2 nm and length of about $70 \mathrm{~nm}$. The studied PA6 nanocomposites became reinforced by very small filler platelets as it is seen from the results of the mechanical tests (Table I, in which the following symbols are used: $E$ - Young's modulus; $\sigma_{\mathrm{b}}$ tensile strength; $\sigma_{\mathrm{p}}$ - yield strength; $\Delta l$ - elongation at break; $M$ - flexural modulus; $u$ - Charpy notched impact strength).

TABLE I

Mechanical characteristics of the studied samples.

\begin{tabular}{l|c|c|c|c|c|c|c}
\hline \hline \multicolumn{1}{c|}{ Sample [wt.\%] } & $\begin{array}{c}\text { Nanocomposite } \\
\text { type }\end{array}$ & $\begin{array}{c}E \\
{[\mathrm{MPa}]}\end{array}$ & $\begin{array}{c}\sigma_{\mathrm{b}} \\
{[\mathrm{MPa}]}\end{array}$ & $\begin{array}{c}\sigma_{\mathrm{p}} \\
{[\mathrm{MPa}]}\end{array}$ & $\begin{array}{c}\Delta l \\
{[\%]}\end{array}$ & $\begin{array}{c}M \\
{[\mathrm{MPa}]}\end{array}$ & $\begin{array}{c}u \\
{\left[\mathrm{~kJ} / \mathrm{m}^{2}\right]}\end{array}$ \\
\hline PA6 (100.0/0.0) & - & 3015 & 49.5 & 83.0 & 81.4 & 2740 & 4.7 \\
PA6/Nf919 (97.5/2.5) & exfoliated & 3437 & 53.2 & 87.9 & 20.3 & 2982 & 2.7 \\
PA6/BZ-COCO $(97.5 / 2.5)$ & exfoliated & 3658 & 60.0 & 93.0 & 13.8 & 3436 & 2.8
\end{tabular}

Wide angle X-ray diffraction studies supplied information presented in Table II.

Incorporation of Nf919 and BZ-COCO into PA6 matrix favored formation of the $\gamma$-form in the nanocomposites and resulted in a decrease in total crystallinity 
TABLE II

Crystallinity and content of each of the crystalline forms in the studied samples.

\begin{tabular}{l|c|c|c|c}
\hline \hline \multirow{2}{*}{ Sample } & Crystallinity & \multicolumn{3}{|c}{ Content [\%] } \\
\cline { 3 - 5 } & {$[\%]$} & $\alpha$-form & $\gamma$-form & amorphous \\
\hline PA6 & 60 & 57 & 3 & 40 \\
PA6/Nf919 (97.5/2.5) & 53 & 31 & 22 & 47 \\
PA6/BZ-COCO (97.5/2.5) & 54 & 19 & 35 & 46
\end{tabular}

of polymer matrix in comparison to the pure PA6. Small angle X-ray difraction revealed the lamellar structure of the nanocomposites and pointed out that the long period in them is smaller than in the pure PA6. It means that the samples of the nanocomposites are more disordered, have greater number density of interfaces. The long period decreased in nanocomposites of higher content of the fillers [10]. It results in the increase in the number density of interfaces with the content of the fillers.

Values of glass temperature, $T_{\mathrm{g}}$, from DSC measurements are shown in Table III.

\section{TABLE III}

Glass temperatures.

\begin{tabular}{l|c}
\hline \hline \multicolumn{1}{c|}{ Sample } & $T_{\mathrm{g}}[\mathrm{K}]$ \\
\hline PA6 $(100.0 / 0.0)$ & $305.7 \pm 0.2$ \\
PA6/Nf919 $(97.5 / 2.5)$ & $309.5 \pm 0.5$ \\
PA6/BZ-COCO $(97.5 / 2.5)$ & $317.9 \pm 0.1$
\end{tabular}

The higher values of $T_{\mathrm{g}}$ for PA6/Nf919 and PA6/BZ-COCO mean the reduced segmental mobility of PA6 chains in nanocomposites and reflect the effect of the fillers.

\section{Positron lifetime characteristics}

Positron annihilation lifetime spectra, measured with a standard fast-fast positron lifetime spectrometer (vacuum $10^{-5} \mathrm{~Pa}$, from room temperature (RT) temperature to $413 \mathrm{~K}$, total number of counts under a spectrum equal to about $\left.2 \times 10^{6}\right)$, were fitted with: two discrete components $\left(\tau_{1}, I_{1} ; \tau_{2}, I_{2}\right)$ and the third one, reflecting the $o$-Ps annihilation by pick-off in spherical holes of free volume $\left.\left(\tau_{3}\right\rangle, I_{3}\right)$. The lognormal distribution of the longest lifetimes $\tau_{3}$ was assumed. The ratio $I_{3} / I_{1}=3$ was settled. Due to analysis with the use of the program Lifetime V.9 [13] the $\left\langle\tau_{3}\right\rangle$ value - the average of $\tau_{3}$, characterizing the distribution of the 


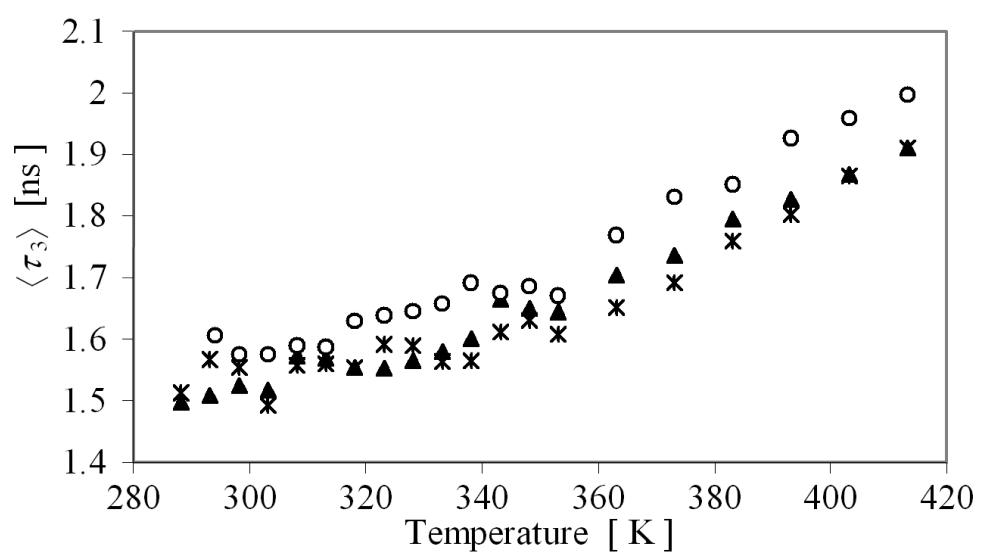

Fig. 1. Values of $\left\langle\tau_{3}\right\rangle$, the average lifetime of the $\tau_{3}$ distribution. Markings are as follows: ^ PA6, $\boldsymbol{\Delta}$ PA6/Nf919 (97.5/2.5), 0 PA6/BZ-COCO (97.5/2.5).

longest lifetimes, and its standard deviation were obtained. In Fig. 1 the $\left\langle\tau_{3}\right\rangle$ values are shown vs. temperature.

The glass transition is only slightly emphasized, while from about $353 \mathrm{~K}$ faster increase in $\left\langle\tau_{3}\right\rangle$ with temperature is clearly seen. According to the literature data [14] the temperature of $353 \mathrm{~K}$ corresponds to the onset of morphological changes of PA6 (disappearance of the denser $\alpha$-form, increase in content and stabilization of the $\gamma$-form).

\section{Free volume distributions}

The mean lifetime $\tau_{3}$ reflects the size of a free volume hole. In our further analysis the model given in $[15,16]$ was used. The drawback of the method is assumption of spherical shape of free volume holes. There do not exist molecular dynamic simulations of free volume for the samples under study. PA6 is a polymer with small fractional free volume $(\mathrm{FFV})$. In literature one can find two values of FFV for PA6 at $T_{\mathrm{g}}: 3.4 \%$ [17] and 6.1\% [18]. It was shown that holes in case of polymers of small total free volume, as PTMSS and PFPDMSS, have shapes close to spherical [6] in opposite to PTMSP (high free volume). Microcavities in the latter, coming from molecular dynamic simulations, are of distinctly elongated shape. Volume distributions of free volume holes for all studied samples were calculated on the basis of the $\tau_{3}$ distributions gained from the positron lifetime spectra measurements. The lifetime and volume distributions are exemplified in Fig. 2a and $\mathrm{b}$ at the highest temperature of PALS measurements.

Values of the average volume $\langle V\rangle$ of free volume holes and their standard deviations SIGMA $_{V}$ are given vs. temperature for all the studied samples in Fig. 3a and $b$. 

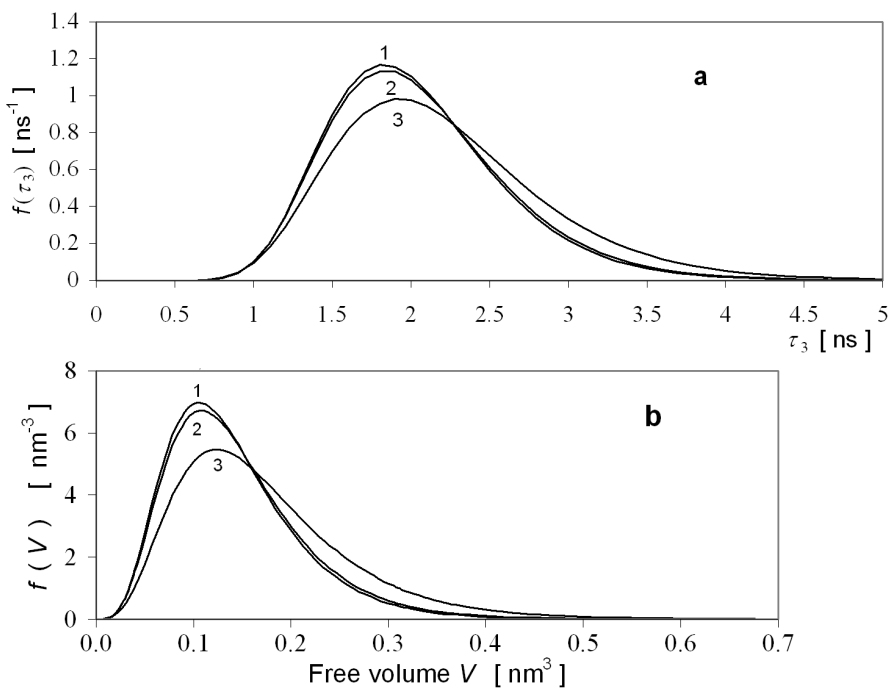

Fig. 2. (a) Distributions of the longest lifetime $\tau_{3}, f\left(\tau_{3}\right)$ at $413 \mathrm{~K}$; (b) volume distributions of the free volume holes, $f(V)$ at $413 \mathrm{~K}$. Markings are as follows: 1 - PA6, 2 — PA6/Nf919 (97.5/2.5), 3 - PA6/BZ-COCO (97.5/2.5).
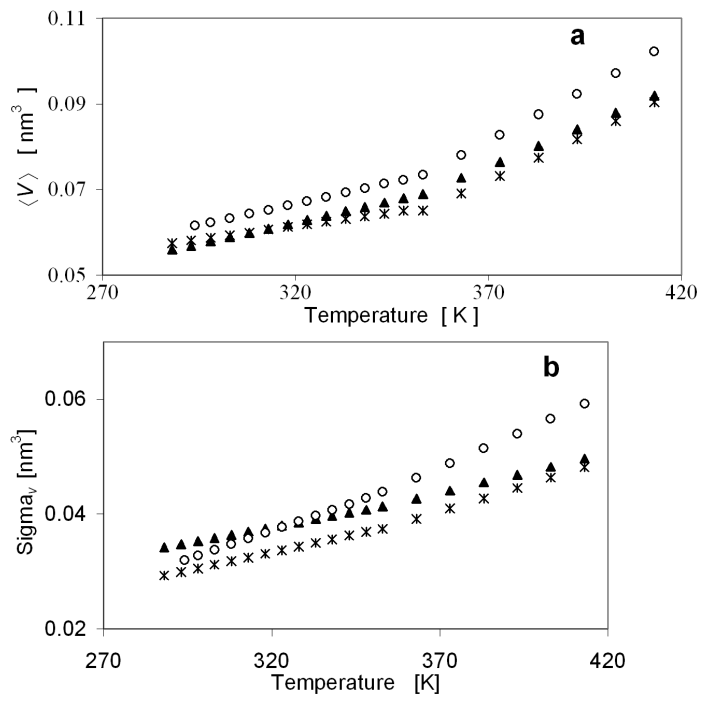

Fig. 3. (a) Average free volume of holes, $\langle V\rangle$; (b) standard deviation, SIGMA $_{V}$, of the distribution of free volume of holes, $f(V)$. Markings are as follows: $\star$ PA6,

PA6/Nf919 (97.5/2.5), 0 PA6/BZ-COCO (97.5/2.5).

\section{Summary and conclusions}

- Ion exchange reactions changed the used pristine bentonites into organobentonites. 
- With the use of polymer melt intercalation method, the organo-bentonites particles were dispersed in the PA6 matrix in a form of individual layers of a 1-2 nm thickness and about $70 \mathrm{~nm}$ length (exfoliated nanocomposites).

- It was an interfacial interaction between PA6 and the silicate that led to the organic and inorganic phases being dispersed at the nanometer level in the studied nanocomposites. The interfacial interaction was facilitated by high aspect ratio of fully dispersed individual layers and resulting from their high surface area.

- The studied PA6 nanocomposites became reinforced by very small filler platelets as it is seen from the results of the mechanical tests.

- Loading of the clay in the PA6 matrix caused increase in the nanocomposite Young and flexural modulus, while elongation at break and Charpy notched impact strength are lower than those recorded for the neat polymer.

- The value of the Young modulus of the nanocomposite PA6/BZ-COCO is higher than the one of PA6/Nf919. This can be attributed to the better surface modification of platelets of BZ-COCO because a higher excess of organic modifier (compared to the ion exchange capacity of BZ-COCO) at the ion exchange stage was used [10].

- Lower elongation at break value for PA6/BZ-COCO nanocomposite is probably due to the hindering effect of the exfoliated organo-bentonite that prevents complete alingment of polymer chains. Due to the effect macromolecules of PA6 are immobilized on the surface of the filler as a result of interaction between the functional groups in PA6 and the organo-cation.

- The higher value of the yielding point characteristic of the nanocomposite PA6/BZ-COCO suggests the presence of better interaction between the polymeric matrix and the organo-bentonite BZ-COCO than in the case of PA6/Nf919. The better interaction was also confirmed by results of Fourier transform IR measurements (changes in thermal characteristics of the amide bands) [10].

- One must take into account that the organo-cation in BZ-COCO contains the polar amide group (-CO-NH-) present also in chains of PA6. Interaction between the amide group from the cation and the one from the chain of PA6 takes place, resulting in formation of hydrogen bonds between the filler and the polymer [10]. It happens evidently at the expense of the $\alpha$-crystalline form contribution to the total crystallinity of the nanocomposite PA6/BZCOCO in which the $\gamma$-crystalline form (less dense) prevails.

- Enhancement of the $\gamma$-form content in nanocomposites is accompanied by decrease in mobility of PA6 chains in them as it is indicated by higher $T_{\mathrm{g}}$ values in them. The statement is supported by increase in $T_{\mathrm{g}}$ values in 
nanocomposites containing more than 2.5 wt.\% of Nf919 and BZ-COCO [10] not studied in the paper.

The highest contribution of the $\gamma$-crystalline form (lesser packing of chains than in the $\alpha$-crystalline form) to the total crystallinity of the sample $\mathrm{PA} 6 / \mathrm{BZ}-\mathrm{COCO}$ and the better interaction between the polymeric matrix and the organo-bentonite BZ-COCO than in the case of PA6/Nf919 (as mentioned above) are in our opinion the main reasons of the clearly different volume distribution of the free volume holes in this sample. The crystalline regions impose topological restrictions on the amorphous regions. Without any doubts the imposed restrictions are lesser in case of the amorphous zones adjacent to the $\gamma$-crystalline form regions. As an effect, we observe in case of the PA6/BZ-COCO sample higher values of $\left\langle\tau_{3}\right\rangle$ and higher contribution of large free volume holes compared to PA6 and PA6/Nf919. Similar influence of the morphology on $o$-Ps characteristics was observed in modified PA6 [19].

- The interpretation given above neglects the possibility of positron annihilation in platelets of the fillers. However, it seems to be justified if there exists "the thick envelope" of PA6 macromolecules around them as it was suggested in [2].

- The interesting and difficult question of positron annihilation mechanism in the studied samples needs further studies of samples with varying content of the fillers, combined with the XRD measurements vs. temperature.

- In opinion of the authors of the paper interfacial regions influence most the positron annihilation mechanism. The statement is based on the observation that the intensity $I_{2}$ of the lifetime component of the $\tau_{2} \approx 350$ ps increased both with the rise of the content of the filler and temperature. In both cases the greater number density of interfaces may happen. Further data will be published later.

\section{Acknowledgments}

The investigation was supported by The State Committee for the Scientific Research under the grant N205 04131/1826 and the PhD ZPORR scholarship program for J. Dołęga as well as by University of Wrocław, grant no. 2016/IFD/07.

\section{References}

[1] S.S. Ray, M. Okamoto, Prog. Polym. Sci. 28, 1539 (2003); E.P. Giannelis, R. Krishnamorti, E. Manias, Adv. Polym. Sci. 138, 107 (2099).

[2] L.A. Utracki, R. Simha, A. Garcia-Rejon, Macromolecules 36, 2114 (2003).

[3] R. Simha, T. Somcynsky, Macromolecules 2, 342 (1969).

[4] R.E. Robertson, in: Computational Modeling of Polymers, Ed. J. Bicerano, Marcel Dekker Inc., New York 1992, p. 301, 304; J. Bicerano, ibidem, p. 397. 
[5] E. Schmidtke, K. Gnther-Schade, D. Hoffman, F. Faupel, J. Mol. Graph. Modelling 22, 309 (2004).

[6] D. Hofmann, M. Heuchel, Yu. Yampolskii, V. Khotimskii, V.P. Shantarovich, Macromolecules 35, 2129 (2002).

[7] D. Hofmann, M. Entrialgo-Castano, A. Lerbret, M. Heuchel, Yu. Yampolskii, Macromolecules 36, 8528 (2003).

[8] C. Nagel, K. Günter-Schade, D. Fritsch, T. Strunskus, F. Faupel, Macromolecules 35, 2071 (2002).

[9] T.D. Fornes, D.R. Paul, Polymer 44, 3945 (2003).

[10] J. Dołęga, Ph.D.Thesis, Wroclaw University of Technology, Wrocław 2007.

[11] G. Wypych, Handbook of Fillers, ChemTec Publishing, Toronto-New York, 1999, p. 43.

[12] A. Viani, A. Gualtieri, G. Artioli, Am. Mineral. 87, 966 (2002); W.E. Worral, Clays and Ceramic Raw Materials, Elsevier Scientific Publishing Company, London 1986 .

[13] J. Kansy, Nucl. Instrum. Methods Phys. Res. A 374, 235 (1996).

[14] D.M. Lincoln, R.A . Vaia, Z. Wang, B. Hsiao, R. Krishnamoorti, Polymer 42, 9975 (2001).

[15] S.J. Tao, J. Chem. Phys. 56, 5499 (1972).

[16] M. Eldrup, D. Lightbody, J.N. Sherwood, Chem. Phys. 65, 51 (1981).

[17] M. Dȩbowska, J. Pigłowski, J. Rudzińska-Girulska, T. Suzuki, Z.Q. Chen, Radiat. Phys. Chem. 68, 471 (2003).

[18] G. Dlubek, F. Redmann, R. Krause-Rehberg, J. Appl. Polym. Sci. 84, 244 (2002).

[19] M. Dȩbowska, J. Pigłowski, C. Ślusarczyk, J. Rudzińska-Girulska, T. Suzuki, R. Yu, Radiat. Phys. Chem. 76, 325 (2007). 